\title{
EL TRABAJO POR PROYECTOS A PARTIR DEL JUEGO POR RINCONES: PLANIFICACIÓN Y PUESTA EN MARCHA DE UN RINCÓN DE RESTAURANTE
}

\author{
Beatriz Martín Sánchez \\ Universidad de Valladolid \\ beamarsan7@hotmail.com \\ Deilis Ivonne Pacheco Sanz \\ Universidad de Valladolid \\ deilisivonne.pacheco@uva.es \\ Alejandro Canedo García \\ Universidad de León \\ acang@unileon.es \\ Yvet Bleye Varona \\ Universidad de Valladolid \\ yvet.b@hotmail.com \\ Angela Gago Morate \\ Universidad de Valladolid \\ agagomorate@gmail.com
}

Recepción Artículo: 13 marzo 2020

Admisión Evaluación: 30 marzo 2020

Informe Evaluador 1: 15 abril2020

Informe Evaluador 2: 19 abril 2020

Aprobación Publicación: 20 abril 2020

\section{RESUMEN}

El juego es uno de los principios metodológicos que se identifica con teorías relacionadas con la corriente constructivista, fundamentada por autores como Vigostky, Ausubel o Piaget, y que guía y enmarca el trabajo por proyectos. El juego, permite al niño/a actuar de forma natural, espontánea, libre, segura, relajada, con posibilidades de error y exenta de crítica. Además, es un elemento fundamental de identidad individual y progresiva pues le permite expresar y compartir ideas, contrastar y poner a prueba conocimientos de sí mismo y de su entorno, pudiéndolos desarrollar y consolidar de manera progresiva. Siendo el juego el principio metodológico y la base del proceso de aprendizaje que orienta la práctica docente de la autora de este trabajo en Educación Infantil, se Ilevó a cabo la modificación del rincón de juego de la casita, que hasta el momento existía, y planificar y poner en marcha un rincón de restaurante. La experiencia se desarrolló con un grupo de 14 alumnos/as de 4 años de una villa pesquera de la costa cántabra. Las situaciones de aprendizaje que se dieron en el aula fueron muy diversas, teniendo como eje central y principal protagonista al niño/a, para garantizar su éxito educativo. El juego de roles que supone la organización de un rincón de restaurante en la clase, llevó a los niños/as a tener un conocimiento de sí mismos cada vez más profundo y completo, a favorecer su autonomía personal, además de tener un mayor conocimiento del entorno cercano y participar activamente en él. Otro de los resultados de la práctica eje- 


\section{EL TRABAJO POR PROYECTOS A PARTIR DEL JUEGO POR RINCONES: PLANIFICACIÓN Y PUESTA EN MARCHA DE UN RINCÓN DE RESTAURANTE}

cutada fue el desarrollo de diferentes tipos de lenguajes para expresar sentimientos, ideas, pensamientos, etc., Ilevando a alcanzar el objetivo final que se pretendía con el proyecto.

Palabras clave: educación infantil; trabajo por proyectos; juego por rincones; participación; éxito educativo

\section{ABSTRACT}

Project work from the game by corners: planning and start-up of a restaurant corner. The game is one of the methodological principles that is identified with theories related to the constructivist current, founded by authors such as Vygotsky, Ausubel or Piaget, and which guides and frames project work. The game allows the child to act naturally, spontaneously, freely, safely, relaxedly, with possibilities for error and without criticism. Furthermore, it is a fundamental element of individual and progressive identity as it allows you to express and share ideas, contrast and test knowledge of yourself and your environment, being able to develop and consolidate them progressively. Being the game the methodological principle and the basis of the learning process that guides the teaching practice of the author of this work in Early Childhood Education, the modification of the play corner of the house, which until now existed, was carried out and planning and launch a restaurant corner. The experience was carried out with a group of 14 students or 4-year-old children from a fishing village on the ntabrian coast. The learning situations that occurred in the classroom were very diverse, having the child as the central axis and main protagonist, to guarantee their educational success. The role play that involves the organization of a restaurant corner in the class, led the children to have an ever deeper and more complete knowledge of themselves, to promote their personal autonomy, in addition to having a greater knowledge of the close environment and actively participate in it. Another result of the practice carried out was the development of different types of languages to express feelings, ideas, thoughts, etc., leading to reaching the final objective that was intended with the project.

Keywords: infant education; project work; corner playing; participation; educational success

\section{ANTECEDENTES DE LA TEMÁTICA A TRATAR}

"Aprende a cocinar, prueba nuevas recetas, aprende de tus errores, no tengas miedo y, sobre todo, diviértete" Julia Child (Cocinera estadounidense)

El trabajo por proyectos contribuye al éxito del proceso de enseñanza-aprendizaje en Educación Infantil y así lo defienden y lo sustentan, con sus teorías, autores clásicos como Vygotsky, Ausubel o Piaget. Asimismo, lo refuerzan y lo corroboran estudios realizados y publicados en los últimos años como, por ejemplo: Mérida, et al. (2011); Ventura (2013); Ureña Borrego (2013); Balcells (2014); Mérida y Ruíz (2016); Ortega Rodríguez (2016); y/o, Pacheco Sanz (2016).

Queda, por tanto, científicamente demostrado que las metodologías de trabajo, que centran todo el proceso de enseñanza-aprendizaje en los niños/as y los convierten en los principales protagonistas, como el trabajo por proyectos, permiten un mayor logro de los objetivos. Sin embargo, el hecho de que el niño/a sea el constructor de su propio aprendizaje, no quiere decir que el rol del maestro/a no sea importante, al contrario, su función será la de acompañar y guiar al alumno/a en este proceso, estar ahí siempre que sea necesario y alentarle para que continúe avanzando en la adquisición de nuevos saberes.

Si queremos que los niños/as sean las piezas claves de todo el proceso educativo, el juego debe ser la base de todo su aprendizaje. En torno al juego se desarrollarán la mayor parte de las actividades del trabajo por proyectos puesto que es algo intrínseco y altamente motivador en sí mismo que el niño/a realiza de forma placentera y gustosa sin obligación de ningún tipo. Esta concepción diferente del niño/a, del maestro/a, de la metodología de trabajo ha hecho cada vez más habitual en las escuelas organizar los espacios de las clases en rincones de juego, en torno a los cuales se desarrollan las situaciones de aprendizaje que tienen lugar en el aula (Laguía, 2008).

Todas las afirmaciones anteriores, se ven claramente reflejadas en la propuesta práctica que se desarrolló, dentro de un contexto educativo real, y que se presenta a continuación. 


\section{OBJETIVOS DE LA INVESTIGACIÓN}

El objetivo de esta investigación está centrado en proponer un modelo de intervención, a través de un ejemplo real, para ejecutar el trabajo por proyectos en un aula de Educación Infantil. Para ello, se definen tanto el objetivo general como los objetivos específicos de dicho modelo.

Objetivo general del proyecto: se centró en planificar y poner en marcha el rincón de un restaurante. Este objetivo, se concretó en una serie de objetivos más específicos, conducentes al desarrollo de competencias, propias de la edad.

- Construir su propia identidad e ir formándose una imagen de sí mismo positiva y ajustada, asumiendo roles vinculados a la cocina y a la hostelería, descubriendo cuáles son sus mejores competencias para ponerlas al servicio del objetivo común del grupo.

- Adquirir autonomía en la realización de sus actividades habituales y en las nuevas que vaya asumiendo como cotidianas en el transcurso de este proyecto (poner la mesa, servir el agua, cortar alimentos, cocinar platos sencillos).

- Practicar hábitos básicos de salud, bienestar y vida sana relacionados con la alimentación.

- Desarrollar su capacidad de iniciativa en la organización de eventos.

- Establecer relaciones sociales satisfactorias en ámbitos cada vez más amplios, incluso con personas ajenas al centro que participan en el desarrollo de este proyecto.

- Observar y explorar su entorno para conocer y comprender la realidad y participar en ella.

- Descubrir en las matemáticas las herramientas necesarias para gestionar algunos aspectos relacionados con los alimentos y el restaurante.

- Representar aspectos de la realidad vivida o imaginada de forma cada vez más personal y ajustada a los diferentes contextos y situaciones desarrollando competencias comunicativas en diferentes lenguajes y formas de expresión.

- Utilizar el lenguaje oral de forma cada vez más adecuada a las diferentes situaciones de comunicación.

- Aproximarse a la lectura y a la escritura en las situaciones de la vida cotidiana y en todo lo relacionado con el restaurante.

Estos objetivos se alcanzaron con el trabajo de contenidos de distintos tipos, tal y como se observa en la tabla 2.

\section{MUESTRA Y/O PARTICIPANTES}

El proyecto de trabajo se contextualizó en el aula de $2^{0}$ nivel de $2^{0}$ ciclo de Educación Infantil, 4 años, de un pequeño colegio de una sola línea situado en una localidad de la costa cántabra en España. El grupo se componía de 14 alumnos/as, 12 niños y 2 niñas. Niños/as enérgicos, deseosos de aprender y participar en todo lo que se les proponía, con destrezas sociales suficientes como para regular mejor su comportamiento, tener sentimiento de pertenencia a un grupo y con capacidad para mantener conversaciones más largas e interesantes, en las que todos/as eran capaces de aportar ideas para beneficio del grupo. Ninguno de los alumnos/as presentaba dificultades de aprendizaje, únicamente existía, entre ellos, diferencias propias de la edad (unos nacidos a principios de año y otros en el último trimestre) y del ambiente familiar, que era muy variado.

Es importante destacar que, para el grupo, esta fue su primera experiencia de trabajar por proyectos y, aunque a las familias se les había explicado en qué consistía la metodología y cómo podían colaborar, en principio se desconocía cuál iba a ser su grado de implicación.

\section{METODOLOGÍA}

El proyecto que se presenta, surge a partir de una asamblea que tiene lugar en septiembre, un lunes a primera hora. Como en todas las asambleas, principalmente después de un fin de semana, todos los niños/as querían contar lo que habían hecho, dónde habían estado, y hacer partícipes a los demás de sus vivencias. En ese momento de intercambio, uno de los niños de la clase cuenta a los demás que había ido a comer una hambur- 


\section{EL TRABAJO POR PROYECTOS A PARTIR DEL JUEGO POR RINCONES: PLANIFICACIÓN Y PUESTA EN MARCHA DE UN RINCÓN DE RESTAURANTE}

guesa a un restaurante de comida rápida. Este hecho suscitó el interés del resto, sobre todo por el regalo que acompaña a los menús infantiles. Esta situación se aprovechó para que la tutora del grupo mostrara interés por la cuestión y preguntara si le había gustado la comida, a lo que el niño respondió que prefería los macarrones que cocina su abuela. Los demás niños/as empezaron a intervenir en la conversación contando lo que les gustaba comer, sus experiencias en restaurantes, etc.

Debido al inicio del curso la clase todavía no estaba del todo organizada, y teniendo en cuenta la conversación que se había mantenido, surgió la idea de proponerles la sustitución del rincón de la casita por un rincón del restaurante. Sin saber muy bien si el proyecto prosperaría, puesto que la casita era uno de los rincones que tenía más éxito el curso anterior, se lanzó la propuesta. Surgió en ese momento el trabajo de lo que sería un maravillo proyecto.

\section{JUSTIFICACIÓN DEL PROYECTO}

Los principios básicos que enmarcaron el trabajo del proyecto, se sustentaron en las directrices marcadas por el D. 79/2008 de 14 de agosto, que se establece en el currículum del 2 Ciclo de Educación Infantil, en la Comunidad Autónoma de Cantabria en España (art. 4: Principios Pedagógicos y Anexo II: Orientaciones Metodológicas).

Colaboración entre la familia y la escuela: Las familias fueron informadas al principio de curso en qué consistía el trabajo por proyectos y cómo era la manera de trabajar. Una vez empezado el proyecto, se les informó debidamente cuál era el objetivo de esta nueva forma de trabajar, y se les dio pautas y estrategias para que ayudaran a lograrlo. De esta manera, se inició un intercambio fluido de información entre ellas y el colegio.

Coordinación entre todos los profesionales del colegio: La presencia simultánea en el aula de diferentes docentes hicieron preciso una coordinación, así como la planificación y reflexión conjunta, la reorganización y la evaluación continua y responsable de los procesos de aprendizaje. Se utilizaron las Reuniones de Ciclo y los Claustros para informar al resto de miembros del equipo educativo del trabajo que se tenía entre manos, asegurando así la coherencia, la continuidad y la toma de decisiones compartida.

Actividad, experimentación y juego: La actividad del niño/a, a través del juego, fue la principal fuente de aprendizaje y desarrollo. El juego de roles que supuso la organización de un rincón de restaurante, los llevó a crear un conocimiento del mundo cercano y a participar activamente en él.

Enfoque comunicativo, sociocultural y funcional: Teniendo en cuenta que el aprendizaje es un proceso cognitivo, pero también una actividad social y cultural, se plantearon situaciones didácticas en las que se hacía real el uso y manejo de textos y mensajes variados (invitaciones para inauguración del restaurante, listas de la compra, etc.).

Socialización de los aprendizajes: Se utilizaron estrategias organizativas, metodológicas y didácticas que hacían posible que todos/as aportarán ideas y que las opiniones de todos/as fueran tenidas en cuenta.

Aprendizaje cooperativo: Se trabajó, principalmente, de forma colectiva (grupo-ciclo, grupo-clase, grupos pequeños, parejas y tríos), porque se tenía claro que el aprendizaje cooperativo no consistía en el hecho de que los niños/as estuvieran sentados juntos, realizando tareas individuales, sino que se produjeran intercambios entre ellos para abordar la tarea con un objetivo común. Todos/as tenían la oportunidad de argumentar, explicar y contrastar información. La aportación de cada uno, desde sus propias posibilidades, era imprescindible para el progreso del grupo. Todos/as colaboraban para poder poner en marcha el restaurante.

Atención a la diversidad: Partiendo de que cada niño era diferente, se potenció positivamente su autoestima ofreciendo apoyo para que cada uno se sintiera capaz y competente, para que fueran conscientes de sus capacidades y limitaciones y para que pudieran pedir ayuda y superar las dificultades con las que se encontraban; se alentó a los niños/as en sus decisiones e intentos mostrándoles el error como parte del logro, fomentándoles su participación e iniciativas; se les brindó variadas posibilidades de elección ofreciéndoles diferentes actividades y experiencias gratificantes y motivadoras; se prestó especial atención a la manifestación de sus emociones dándoles múltiples oportunidades de manifestarlas y expresarlas. 
Integración de los conocimientos informales: Este proyecto surgió de la experiencia de un niño en un restaurante y de conocimientos informales sobre la realidad que tenía (hipótesis provisionales, conceptos incompletos, procedimientos rutinarios). Conocimientos que fueron detectados y aprovechados como punto de partida de los aprendizajes posteriores. Espontáneamente, los niños/as se plantearon hipótesis que, en ocasiones, no eran completas 0 estaban teñidas de fantasía; pero, reales o no, fueron el punto de arranque a partir del cual se construyeron nuevos conocimientos.

Es lo que se conoce como ideas previas y, para detectar las relacionadas con este proyecto, se aprovechó la asamblea de inicio del curso para plantear preguntas como: ¿habéis estado alguna vez en un restaurante?; ¿Qué habéis comido? Luego, se formuló la hipótesis de: si yo fuera a un restaurante... ¿qué tendría que hacer? Algunas de las hipótesis, teñidas de respuestas espontáneas, se muestran en la figura 1.

Figura 1. Extracto de una conversación mantenida en una asamblea de clase

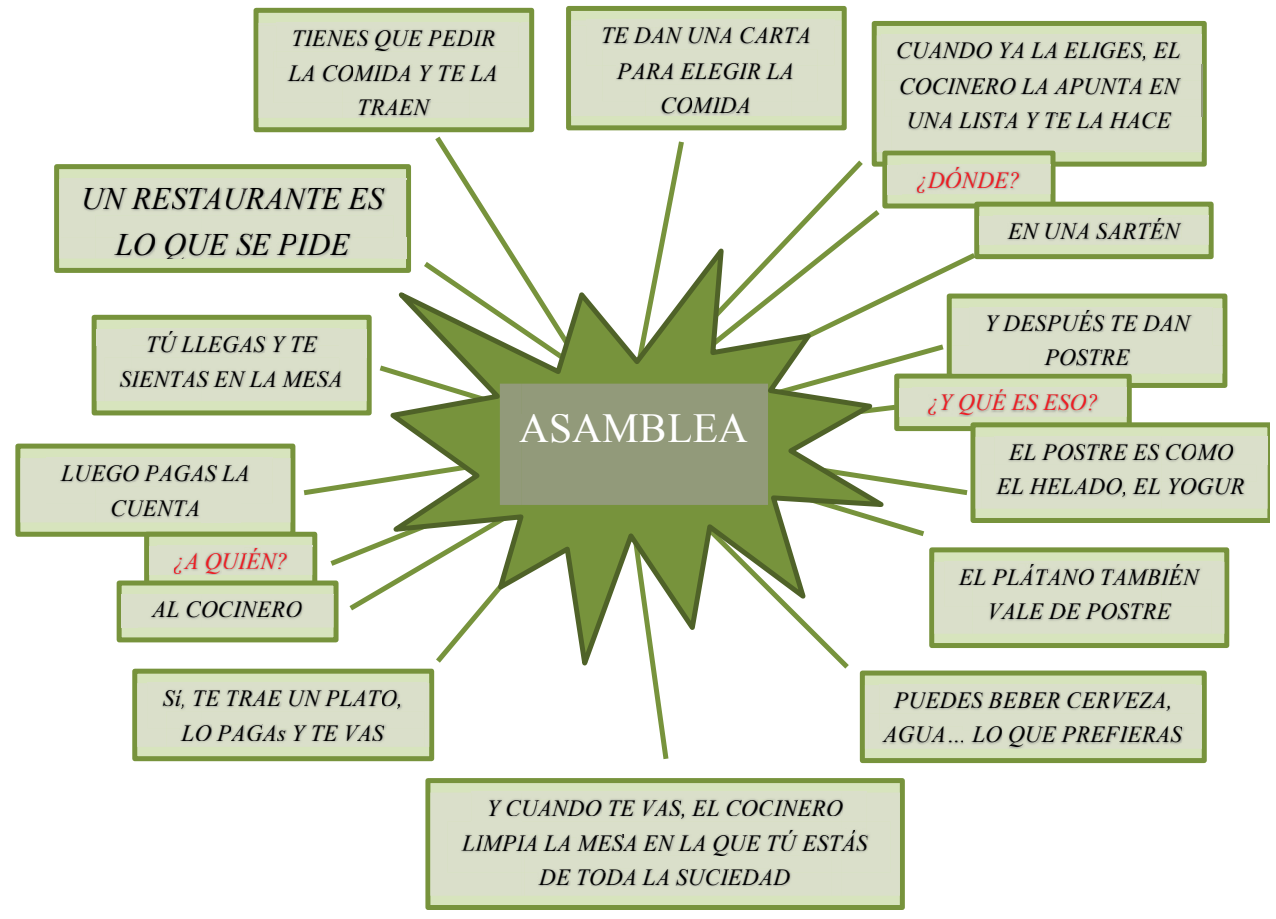

\section{RESULTADOS ALCANZADOS}

Los objetivos propuestos para el desarrollo del proyecto, se fueron alcanzando a partir de situaciones de aprendizaje que tuvieron lugar en el aula.

Durante el tiempo que duró el proyecto se realizaron numerosas actividades: algunas más dirigidas, otras más libres, algunas desarrolladas en gran grupo, otras en pequeño grupo, unas en parejas y otras de forma individualizada (Martín, 2018).. Unas actividades han precisado de materiales específicos, otras actividades de materiales más comunes. Pero todas ellas llevaron a conseguir al final del proyecto el objetivo principal, que era, la puesta en marcha de un rincón de restaurante.

Algunas de las actividades que destacaron por su interés, atractivo o relevancia, se muestran en la tabla 1 


\section{EL TRABAJO POR PROYECTOS A PARTIR DEL JUEGO POR RINCONES: PLANIFICACIÓN Y PUESTA EN MARCHA DE UN RINCÓN DE RESTAURANTE}

\section{Tabla 1.Situaciones de aprendizaje: actividades desarrolladas}

ACTIVIDAD DESCRIPCIÓN

Elegir nombre

Repartir roles

Conseguir estrellas

Elaborar el menú

Visita a una escuela de hostelería
Después de realizar una lista con los nombres de los restaurantes que los niños/as conocen y hablar sobre ellos, era el momento de ponerle nombre al futuro restaurante. Algunos niños/as se atrevieron a señalar algún nombre que les gustaba, pero como no llegan a ningún acuerdo, puesto que cada uno defiende su nombre como el mejor, se realizó una votación. Al finalizar dicha votación, contar los votos y ver cuál era el que más tenía, el nombre ganador fue CHIMENEO. A partir de esta elección tenían que realizar varias tareas: Elaborar el cartel para el restaurante, realizar un logotipo para el cartel y las cartas de los menús...

En una de las asambleas se habló sobre las personas que están un restaurante. Se habló de cocineros/as, camareros/as, clientes, de cuáles eran las funciones de cada uno de ellos. También se les explicó la labor de otras personas que trabajan en un restaurante: chef, metre, sumiller. Eligieron el rol que cada uno quería desempeñar en el restaurante y se prepararon para ser los mejores en cada uno de los papeles elegidos,

Estudiando alguno de los cocineros más conocidos (cada niño/a preparó, junto a su familia, una pequeña exposición sobre un cocinero para presentárselo al resto de compañeros). Vieron que muchos de ellos tenían en sus restaurantes estrellas Michelin. El Restaurante Chimeneo no va a ser menos, así que prepararon estrellas, catorce, una por niño/a.

A partir de las cartas de diferentes restaurantes que fueron llegando a la clase, vieron la variedad de menús que éstos ofrecían. Clasificaron los platos en primeros, segundos y postres, así elaboraron distintos menús para ofrecerles a los clientes y confeccionaron su propia carta.

Se aprovechó, que en uno de los Institutos de la localidad donde está el centro escolar se imparte un módulo de hostelería, para visitar sus instalaciones. Allí tanto alumnos/as como profesores/as dieron algunas ideas que se podían utilizar en el restaurante, cocinaron con ellos unos de los alimentos típicos de la gastronomía cántabra, el sobao pasiego, compartieron experiencias y participaron en un desayuno conjunto. 
Cocinar algunas recetas

Visita de un barman

\section{Montar y desmontar servicios}

Decorar la vajilla y las paredes del restaurante

Inauguración
Después de conocer diferentes utensilios de cocina (los niños/as trajeron a la clase los más diversos utensilios: lenguas, rayadores, separadores de yemas, tamices) y cómo se organizaban los alimentos dentro de la pirámide alimentaria (a partir de revistas de propaganda de diferentes supermercados), era hora de ponerse a cocinar, así se elaboraron diferentes recetas de cocina.

El papá de una de las niñas de la clase trabajaba como barman en un hotel y vino al cole a enseñar los objetos con los que trabajaba y cómo se hacían cócteles y smoothies. Los probaron y les encantaron

Como buenos metres aprendieron a montar y desmontar las mesas: poner los manteles, colocar cada elemento en su lugar, servir con jarras.

Se utilizaron mandalas redondos para decorar la vajilla y se realizaron bodegones, siguiendo las ideas de algunos pintores, para ambientar las paredes del restaurante

Tras preparar todo lo necesario para la puesta en marcha de un restaurante en la clase, llegó el gran día de la inauguración. En este día tan especial, todo debía estar organizado al detalle: invitaciones, canapés, cinta para cortar, guardia de seguridad.

Fuente: Elaboración propia.

Asimismo, se alcanzaron los objetivos específicos, conducentes al desarrollo de competencias, propias de la edad, a partir del abordaje de los contenidos educativos y a través de actividades globalizadas relacionadas con: Lengua Extranjera, Música y Psicomotricidad. Contenidos que se recogen en la tabla 2. 


\section{EL TRABAJO POR PROYECTOS A PARTIR DEL JUEGO POR RINCONES: PLANIFICACIÓN Y PUESTA EN MARCHA DE UN RINCÓN DE RESTAURANTE}

Tabla 2. Contenidos trabajados a través de actividades globalizadas

\begin{tabular}{|c|c|c|}
\hline $\begin{array}{c}\text { Conocimiento de sí mismo } \\
\text { y autonomía personal }\end{array}$ & Conocimiento del entorno & $\begin{array}{c}\text { Lenguaje: Comunicación y } \\
\text { Representación }\end{array}$ \\
\hline Trabajo en equipo. & Utensilios de la cocina y & Expresión de ideas y \\
\hline $\begin{array}{l}\text { Fomento de la } \\
\text { responsabilidad en trabajo }\end{array}$ & $\begin{array}{l}\text { de la mesa: funciones y } \\
\text { usos. }\end{array}$ & $\begin{array}{l}\text { argumentación. } \\
\text { Participación activa en }\end{array}$ \\
\hline en equipo. & Conocimiento de los & actividades \\
\hline Juego cooperativo. & diferentes alimentos y su & comunicación oral. \\
\hline $\begin{array}{lrr}\text { Hábitos } & \text { sociales } & \text { de } \\
\text { cortesía: } & \text { saludar } & \text { y }\end{array}$ & Discriminación & $\begin{array}{l}\text { Respeto del turno de } \\
\text { palabra. }\end{array}$ \\
\hline despedirse. & $\begin{array}{l}\text { alimentos a través de los } \\
\text { sentidos. }\end{array}$ & Vocabulario relacionado \\
\hline Hábitos alimenticios. & Oficios asociados al & $\begin{array}{l}\text { con la almmentacion y los } \\
\text { restaurantes. }\end{array}$ \\
\hline $\begin{array}{l}\text { Normas básicas de } \\
\text { comportamiento en la } \\
\text { mesa. }\end{array}$ & $\begin{array}{l}\text { restaurante. } \\
\text { Cualidades de los objetos } \\
\text { y materiales. }\end{array}$ & $\begin{array}{l}\text { Manejo de textos de uso } \\
\text { social: recetarios, } \\
\text { periódicos, revistas, folletos }\end{array}$ \\
\hline Búsqueda de soluciones: & Atención a las medidas. & de propaganda \\
\hline $\begin{array}{l}\text { Cooperación en la solución } \\
\text { de conflictos. }\end{array}$ & & Elaboración de textos \\
\hline $\begin{array}{l}\text { Sentido del gusto, vista, } \\
\text { olfato, y tacto. }\end{array}$ & $\begin{array}{l}\text { Cuantificadores: más que, } \\
\text { menos que, igual que. }\end{array}$ & $\begin{array}{l}\text { menús, cartas, carteles, } \\
\text { invitaciones. }\end{array}$ \\
\hline $\begin{array}{l}\text { Percepción a través de los } \\
\text { sentidos. }\end{array}$ & $\begin{array}{l}\text { Percepción de las partes de } \\
\text { un todo. }\end{array}$ & $\begin{array}{l}\text { Obras pictóricas: } \\
\text { bodegones. }\end{array}$ \\
\hline $\begin{array}{l}\text { Identificación y control del } \\
\text { apetito. }\end{array}$ & $\begin{array}{l}\text { Organización y } \\
\text { estructuración de espacios. }\end{array}$ & $\begin{array}{l}\text { Percepción de las partes de } \\
\text { un todo. }\end{array}$ \\
\hline $\begin{array}{l}\text { Control sobre la postura y } \\
\text { el equilibrio. }\end{array}$ & & \\
\hline Organización de eventos. & & \\
\hline
\end{tabular}

Fuente: Elaboración propia.

En cuanto a la evaluación realizada a lo largo de todo el proyecto de trabajo, la misma fue global y continua, dando la oportunidad de rectificar errores, modificar actividades en función de las dificultades encontradas e ir adaptando el proyecto a las necesidades e intereses de los alumnos y dar coherencia a todo el proyecto. Los instrumentos de evaluación utilizados: diario de aula, observación directa y sistemática, dossier de trabajo individual y organización de la inauguración del restaurante, dieron información sobre los resultados alcanzados con el desarrollo del proyecto, vinculados a los principios metodológicos que lo fundamentan (ver tabla 3). 
Tabla 3. Aspectos a evaluar y resultados

\begin{tabular}{|c|c|}
\hline $\begin{array}{l}\text { ASPECTOS A } \\
\text { EVALUAR }\end{array}$ & RESULTADOS \\
\hline Motivación del alumnado & $\begin{array}{l}\text { El alumnado mostró una gran motivación durante todo el } \\
\text { proyecto, de hecho, se amplió la duración del mismo } \\
\text { aprovechando esta motivación y se incorporaron nuevos } \\
\text { contenidos de aprendizaje; la implicación de los alumnos } \\
\text { y su grado de participación fue variando dependiendo de } \\
\text { la actividad que se desarrollaba, pero, en líneas } \\
\text { generales, fue buena. }\end{array}$ \\
\hline Actitud de la maestra & $\begin{array}{l}\text { El papel que ejerció fue de mediadora, interviniendo } \\
\text { siempre que fue necesario, pero no interfiriendo en los } \\
\text { aprendizajes realizados por los niños/as, dejándoles que } \\
\text { ellos fueran los protagonistas con actividades } \\
\text { manipulativas, expositivas... }\end{array}$ \\
\hline El proyecto & $\begin{array}{l}\text { Aunque en un principio el proyecto se inició como parte } \\
\text { de la organización de la clase, después se desarrolló } \\
\text { como un proyecto de trabajo en toda regla. }\end{array}$ \\
\hline $\begin{array}{c}\text { Materiales y recursos } \\
\text { utilizados }\end{array}$ & $\begin{array}{l}\text { Muchos de los materiales e instrumentos utilizados } \\
\text { fueron aportados por las familias, por lo que eran } \\
\text { materiales cercanos a los niños/as. }\end{array}$ \\
\hline $\begin{array}{l}\text { Participación de los } \\
\text { diferentes sectores de la } \\
\text { Comunidad Educativa }\end{array}$ & $\begin{array}{l}\text { La participación de los distintos miembros de la } \\
\text { Comunidad Educativa fue una realidad en todo el } \\
\text { proyecto acercando la escuela al entorno. Se les necesitó } \\
\text { para la recogida de información, para la ayuda en } \\
\text { determinadas tareas, para la explicación de diferentes } \\
\text { oficios y se les invitó en la inauguración del restaurante. }\end{array}$ \\
\hline
\end{tabular}

Fuente: Elaboración propia.

\section{DISCUSIÓN Y CONCLUSIONES}

La propuesta llevada a cabo dentro del aula, demostró cómo el juego de roles que los niños interpretaron en el rincón de restaurante, y su trabajo a partir de un proyecto, permitió el desarrollo global y la construcción y dinamización de un conocimiento más elaborado. Este proyecto permitió la realización de actividades didácticas significativas en las que el alumnado pudo explorar, investigar, y reflexionar sobre su propia identidad, sobre los demás y sobre el entorno que le rodeaba, proporcionándole más oportunidades y opciones de aprendizaje.

El trabajo por proyectos desarrollado, dio respuesta a preguntas claves dentro del proceso educativo, por ejemplo: ¿Qué objetivos generales se quieren conseguir?, ¿Qué objetivos didácticos y competencias específicas alcanzará el alumnado?, ¿Qué contenidos se van a trabajar?, ¿Qué actividades se plantearán? ¿Cómo implicar a las familias?, ¿Cómo realizar la evaluación?, ¿Qué instrumentos utilizar para la evaluación?

\section{REFERENCIAS BIBLIOGRÁFICAS}

Balcells, M. (2014). El trabajo por proyectos: una metodología global. Madrid: Cuadernos de Pedagogía, Wolters Kluwer, No. 450.

Decreto 78/2008, de 14 de agosto por el que se establece el currículo del segundo ciclo de Educación Infantil en

la Comunidad Autónoma de Cantabria.

Laguía, M.J. (2008). Rincones de actividad en la escuela infantil (0 a 6 años). Barcelona: Graó. 


\section{EL TRABAJO POR PROYECTOS A PARTIR DEL JUEGO POR RINCONES: PLANIFICACIÓN Y PUESTA EN MARCHA DE UN RINCÓN DE RESTAURANTE}

Martín Sánchez, B. (2018). El trabajo por proyectos en Educación Infantil: Una experiencia real en el aula. (Trabajo Fin de Grado). Universidad de Valladolid, España. Recuperado de: http://uvadoc.uva.es/handle/10324/33051

Mérida, R., Barranco, B., Criado, E., Fernández, N., López, R.M. y Pérez, I. (2011). Aprender investigando en la escuela y la universidad. Una experiencia de investigación- acción a partir del Trabajo por Proyectos. Investigación en la escuela, 73, 65-76.

Mérida Serrano, R. y Ruiz Torres, M. C. (2016). Promover la inclusión de las familias a través del desarrollo de Proyectos de Trabajo. Un estudio de caso. Revista Complutense de Educación, 27(3), 943-961. D0I: 10.5209/rev_RCED. 2016.V27. N3.47022.

Ortega Rodríguez, P.J. (2016). Los trabajos por proyectos en Educación Infantil. En primera persona: estudio de caso biográfico-narrativo de una maestra. Investigación en la Escuela, (88), 112-116. Recuperado de www.investigacionenlaescuela.es/articulos/R88/R88-7.

Pacheco Sanz, D. I. (2016). Desarrollo del proceso de enseñanza-aprendizaje basado en las Inteligencias Múltiples. Material Docente del Máster en Inteligencias Múltiples aplicadas en la Educación Secundaria. Universidad de Alcalá, Fundación General de la Universidad de Alcalá y OFINET S.L.

Ureña Borrego, M. (2013). Competencias y proyectos en Educación Infantil: hacia una educación holística. Calanda: Revista didáctica de la acción educativa española en Francia, (8), pp. 22-31.

Ventura, M. (2013). La perspectiva de los proyectos de trabajo como un motor de cambio en la organización del currículum y en la gestión del tiempo y el espacio. Investigación en la escuela, 79,19-30. 